\title{
Atualização das normas brasileiras para o International Affective Picture System (IAPS)
}

\author{
Update of the Brazilian norms for the International Affective Picture System (IAPS)
}

\author{
Cristina Lasaitis ${ }^{1}$, Rafaela Larsen Ribeiro ${ }^{2}$, Marcelo Ventura Freire ${ }^{3}$, Orlando Franscisco Amodeo \\ Bueno $^{4}$
}

${ }^{1}$ Mestranda, Programa de Pós-Graduação em Psicobiologia, Universidade Federal de São Paulo - Escola Paulista de Medicina (UNIFESP-EPM), São Paulo, SP. ${ }^{2}$ Doutora. Professora adjunta, Departamento de Psicobiologia, UNIFESP-EPM. ${ }^{3}$ Pós-doutor. Professor, Escola de Artes, Ciências e Humanidades, Universidade de São Paulo (USP), São Paulo, SP. ${ }^{4}$ Livre-docente. Professor adjunto, Programa de Pós-Graduação em Psicobiologia, UNIFESP-EPM. Apoio financeiro: FAPESP (processo $n^{\circ}$ 2006/05144-7) e AFIP.

\section{Resumo}

Este trabalho expande a normatização brasileira do International Affective Picture System (IAPS), um instrumento de imagens afetivas, amplamente utilizado em pesquisas, com valores normativos para 240 novos estímulos, e confirma as categorias de imagens "agradáveis", "neutras" e "desagradáveis". Também são apresentados os resultados comparando os padrões de respostas de homens e mulheres. No total, participaram 448 estudantes universitários brasileiros (179 homens e 269 mulheres), que avaliaram as fotografias em termos de prazer, alerta e dominância, através da escala Self Assessment Manikin (SAM). O procedimento adotado foi o mesmo de outro estudo já desenvolvido para as normas norte-americanas.

Descritores: IAPS, emoção, diferenças sexuais, prazer, alerta.

\begin{abstract}
This study includes the standardization of 240 new stimuli in the Brazilian norms for the International Affective Picture System (IAPS), an instrument of affective images widely used in research, and confirms the validity of the categories of "pleasant," "neutral" and "unpleasant" pictures. A comparison was performed of the scores of affective ratings between the male and female samples of university students (179 males, and 269 females), who assessed the affective content of photographs in terms of valence, arousal and dominance using the Self Assessment Manikin (SAM) scale. The methods were the same used in a study conducted for the American norms.
\end{abstract}

Keywords: IAPS, emotion, sex differences, valence, arousal.

\section{Introdução}

O International Affective Picture System (IAPS) ${ }^{1}$ é um instrumento de imagens afetivas que abordam diferentes eventos da vida e valores veiculados pela mídia presentes na cultura geral de muitos países (como família, esporte, sexualidade, violência, saúde, etc.). Por ser um instrumento eficiente na indução de diferentes estados emocionais e permitir grande nível de controle dos parâmetros de exposição e mensuração em contextos experimentais, o IAPS tem sido amplamente utilizado em pesquisas na área da cognição, do afeto, do comportamento, das atitudes sociais, da psicofisiologia, entre outras, tanto para estudar pacientes com distúrbios neuropsiquiátricos quanto populações saudáveis.

Estudos têm demonstrado que os principais fatores implicados na experiência emocional são o prazer e o

\section{Correspondência:}

Cristina Lasaitis, Departamento de Psicobiologia, UNIFESP-EPM, Rua Botucatu, 862, $1^{\circ}$ andar, CEP 04023-062, São Paulo, SP. Tel. (11) 2149.0155/182, Fax (11) 5572.5092. E-mail: cristinalasaitis@gmail.com.

Não há conflitos de interesse associados à publicação deste artigo.

Copyright (C) Revista de Psiquiatria do Rio Grande do Sul - SPRS 
alerta, que, juntos, constituem um espaço afetivo representativo da valência hedônica e da ativação motivacional ${ }^{2}$. Nesse espaço bidimensional, os julgamentos de prazer e desprazer refletem o sistema apetitivo-aversivo, enquanto a avaliação do alerta indica a intensidade de ativação motivacional ${ }^{3}$. As figuras do IAPS se distribuem através desse espaço afetivo de prazer e alerta, podendo ser categorizadas, de acordo com os estados psicofisiológicos que produzem, em: imagens agradáveis alertantes, agradáveis relaxantes, neutras ou desagradáveis ${ }^{4}$. Curiosamente, o instrumento não dispõe de imagens desagradáveis de baixo alerta. Uma terceira dimensão, dominância, reflete o nível de controle da resposta emocional (cujo espectro varia de submisso a dominante), e também tem sido comumente usada para avaliar os estímulos do IAPS.

Para a classificação subjetiva dos estímulos do IAPS, é usado o Self Assessment Manikin ${ }^{5}$, um instrumento de auto-relato composto por três escalas, sendo uma para cada dimensão estudada (prazer, alerta e dominância). Cada dimensão é retratada por cinco desenhos que simbolizam diferentes gradações emocionais, por exemplo: a dimensão prazer é representada por um boneco triste/descontente em um extremo e um alegre/sorridente em outro, e suas respectivas gradações estão intercaladas entre esses extremos. Os sujeitos respondem às fotografias marcando um X sobre um dos cinco desenhos em cada escala ou nos espaços entre eles, resultando em nove possibilidades de resposta. O valor mais baixo para cada dimensão é 1, que indica o escore mais baixo de prazer, alerta ou dominância, enquanto 9 é o valor máximo e reflete o nível mais elevado das mesmas dimensões. O SAM é um instrumento de fácil utilização e pode ser usado para aferir respostas emocionais a variados estímulos e em diferentes tipos de sujeitos experimentais.

Em humanos, a experiência emocional não pode ser reduzida ao determinismo biológico, como um produto integral dos circuitos motivacionais, mas é afetada por outros fatores, sejam de ordem pessoal, social, cultural ou situacional. Não obstante, o caráter estereotipado das emoções através de inúmeras línguas e diferentes contextos socioculturais corrobora a hipótese de uma determinação biológica universal. Disso resulta que, guardadas pequenas diferenças, o padrão de resposta aos estímulos do IAPS, através das dimensões de prazer e alerta, tem se mostrado muito semelhante nas diferentes populações estudadas, como pode ser observado quando são comparados os valores das normatizações americana ${ }^{1}$ e espanhola ${ }^{6,7}$ com a brasileira $^{8,9}$. O IAPS é um instrumento em constante desenvolvimento e, desde seu lançamento, tem sido acrescido de novos conjuntos de fotografias. A padronização brasileira, iniciada por Ribeiro et al. ${ }^{8,9}$, incluiu 707 estímulos referentes aos conjuntos 1 a 12 do IAPS.

Os objetivos do presente estudo foram: 1) expandir a padronização brasileira do IAPS aos 240 estímulos mais recentes, que compõem os conjuntos 13 a 16 do estudo original; 2) comprovar a validade das categorias de imagens; 3) comparar os diferentes padrões de resposta emocional entre homens e mulheres brasileiros.

\section{Método}

\section{Sujeitos}

Participaram do estudo 448 estudantes universitários (179 homens e 269 mulheres), de idades entre 18 e 62 anos (média $24,2 \pm 7,8$ ), que têm o português como idioma nativo, selecionados de diferentes cursos (medicina, biomedicina, psicologia, ciências contábeis, administração e educação física) de universidades públicas e privadas da cidade de São Paulo. A seleção foi feita através de contatos préestabelecidos com professores das faculdades, que permitiram o uso das salas de aula e dos equipamentos de exibição para que os experimentos fossem realizados em turmas. Não foram colhidas informações sobre a condição física ou mental dos sujeitos da pesquisa, contudo, os dados dos voluntários que não tinham visão corrigida, não reportaram seu sexo ou foram negligentes no preenchimento das escalas foram excluídos da análise, representando um total de 9,3\% dos participantes.

\section{Material}

Foram utilizadas 240 fotografias referentes aos conjuntos 13 a 16 do IAPS, originalmente descritas por Lang et al. ${ }^{1}$.

\section{Procedimento}

Os procedimentos empregados e as análises dos resultados seguiram o modelo do estudo anterior ${ }^{8,9}$ que, por sua vez, foi baseado no estudo original ${ }^{1}$. Em cada sessão experimental, foram apresentadas 30 fotografias escolhidas aleatoriamente dos conjuntos 13 a 16 do IAPS. Para que os sujeitos fizessem a classificação dos estímulos no espaço afetivo, foi usada uma versão impressa da escala SAM (versão em papel e caneta) $)^{5}$ que os permitiu classificar cada fotografia em três escalas de 9 pontos, sendo elas: prazer (variando de 1 para agradável a 9 para desagradável), alerta (variando de 1 para calmo/relaxado a 9 para 
agitado/alerta) e dominância (variando de 1 para submisso a 9 para dominante).

Cada sessão experimental incluiu uma projeção de slides contendo instruções e exemplos de pontuações da escala SAM para cada dimensão (prazer, alerta e dominância). Além das 30 fotografias do IAPS classificadas em cada sessão, foram incluídas três fotografias de exemplos utilizadas por Lang, exibidas previamente para que os sujeitos as classificassem em uma folha teste.

Antes de cada sessão, foi feita uma apresentação preliminar com slides de instruções e exemplos de classificações nas dimensões prazer, alerta e dominância. Durante o experimento, uma tela de preparação apresentava o número do slide (de 1 a 30) durante 5 segundos, após esse tempo, uma figura era apresentada durante 5 segundos e, nos 15 segundos subseqüentes, nenhuma figura era apresentada, para que os sujeitos fizessem suas classificações na folha impressa. Depois disso, o experimentador anunciava em voz alta o próximo slide e surgia uma nova tela de preparação. As sessões foram realizadas em salas de aula contendo de 13 a 60 alunos e iluminação adequada para a visualização de slides.

\section{Análise dos dados}

Foram determinadas as médias e os desvios padrão das avaliações afetivas de cada fotografia para todos os sujeitos e, separadamente, para homens e mulheres em cada dimensão. Uma vez que a distribuição das fotografias, dentro dos oito conjuntos estudados, foi feita de forma aleatória, os valores das figuras foram reorganizados de acordo com os conjuntos do estudo original, durante a análise estatística. Foram realizados testes $t$ de Student para comparar as médias obtidas de homens e mulheres para cada figura estudada. O nível de significância adotado foi de $5 \%$.

Adicionalmente à classificação das figuras como agradáveis, neutras e desagradáveis, conforme o trabalho de Ribeiro et al. ${ }^{8}$, foi realizada uma análise de cluster (ou de aglomerados) para confirmar se a adequação dessas categorias é corroborada pela percepção das figuras pelos respondentes expressa nos dados.

\section{Resultados}

Os valores obtidos nas classificações mostram consistência com os dados do primeiro estudo ${ }^{8,9}$. Mulheres, quando comparadas aos homens, atribuíram menores valores de prazer e maiores de alerta para o total das fotografias, mas somente na dimensão dominância houve diferenças significativas, tendo elas reportado valores menores do que os homens (Tabela 1). Outro resultado relevante foi que não houve unanimidade entre os sexos nas fotografias classificadas como agradáveis (A - prazer $>6$ ), desagradáveis (D prazer $<4)$ e neutras $(\mathrm{N}$ - prazer entre 4 e 6$)$, para as quais se verificou que as mulheres classificaram menos figuras como neutras e mais figuras como agradáveis e desagradáveis em relação aos homens (Tabela 2). Dentro dessas classificações, não foram encontradas diferenças nas médias de prazer, alerta e dominância para fotografias neutras e agradáveis. Contudo, para as fotografias consideradas desagradáveis, houve diferenças significativas nas três dimensões, nas quais as mulheres reportaram menores valores de prazer e dominância e maiores valores de alerta.

$\mathrm{Na}$ análise de cluster, considerando simultaneamente as médias masculinas e femininas de prazer, alerta e dominância, de início, foram detectados como claramente distintos apenas um cluster mais homogêneo e um grande cluster mais heterogêneo com grande variabilidade interna, característica de clusters não muito bem separados, embora detectados como um único cluster (Figura 1). O índice $\mathrm{ETA}^{2}$ de proporção de variabilidade interna explicada sugere que no mínimo três clusters são necessários para

Tabela 1 - Médias e desvios padrão das classificações brasileiras para todas as 240 figuras que compõem os conjuntos 13 a 16 do IAPS para as dimensões prazer, alerta e dominância

\begin{tabular}{lcccc}
\hline & \multicolumn{2}{c}{ Homens $(\mathbf{n}=\mathbf{1 7 9})$} & \multicolumn{2}{c}{ Mulheres $(\mathbf{n}=\mathbf{2 6 9})$} \\
\cline { 2 - 5 } & Média & DP & Média & DP \\
\hline Prazer & 5,26 & 2,20 & 5,03 & 2,37 \\
Alerta & 5,42 & 1,66 & 4,47 & 1,88 \\
Dominância & $5,21^{*}$ & 1,21 & 4,94 & 1,56 \\
\hline
\end{tabular}

$\mathrm{DP}=$ desvio padrão.

$* \mathrm{p}<0,05$. 
Tabela 2 - Médias das classificações brasileiras para todas as 240 figuras que compõem os conjuntos 13 a 16 do IAPS para as dimensões prazer, alerta e dominância, para fotografias agradáveis $(\mathrm{A}-$ prazer $>6)$, desagradáveis $(\mathrm{D}-$ prazer $<4)$ e neutras $(\mathrm{N}$ prazer entre 4,0 e 6,0 )

\begin{tabular}{lcccccc}
\hline & \multicolumn{3}{c}{ Homens } & \multicolumn{3}{c}{ Mulheres } \\
\cline { 2 - 7 } & $\mathbf{A}(\mathbf{n}=\mathbf{8 8})$ & $\mathbf{N}(\mathbf{n}=\mathbf{7 4})$ & $\mathbf{D}(\mathbf{n}=\mathbf{7 8})$ & $\mathbf{A}(\mathbf{n}=\mathbf{8 7})$ & $\mathbf{N}(\mathbf{n}=\mathbf{6 4})$ & $\mathbf{D}(\mathbf{n}=\mathbf{8 9})$ \\
\hline Prazer & 7,32 & 5,05 & $2,49^{*}$ & 7,41 & 5,10 & 2,22 \\
Alerta & 4,05 & 5,08 & $7,16^{*}$ & 3,81 & 5,05 & 7,50 \\
Dominância & 6,05 & 5,26 & $3,82^{*}$ & 6,23 & 5,20 & 3,11 \\
\hline
\end{tabular}

$* \mathrm{p}<0,05$.

explicar pelo menos $80 \%$ da variabilidade presente nos dados, e o índice PRE de redução proporcional de variabilidade interna sugere que não haverá grande ganho marginal em utilizar mais que quatro clusters (Figura 2). As médias dos três clusters (Figura 3) indicam que o primeiro cluster (que é o mais claramente distinto dos outros; $\mathrm{n}=79$ ) pode ser identificado como o das figuras desagradáveis, que o segundo cluster $(\mathrm{n}=97)$ é o das figuras neutras, e que o terceiro $(n=64)$ é o das figuras agradáveis.

Os valores normativos da padronização brasileira das figuras que compõem os conjuntos 13 a 16 do IAPS estão disponíveis no site da Universidade federal de São Paulo (UNIFESP), juntamente com os dados obtidos no primeiro estudo, referente aos conjuntos 1 a 12: www.unifesp.br/dpsicobio/adap/adapta.htm.

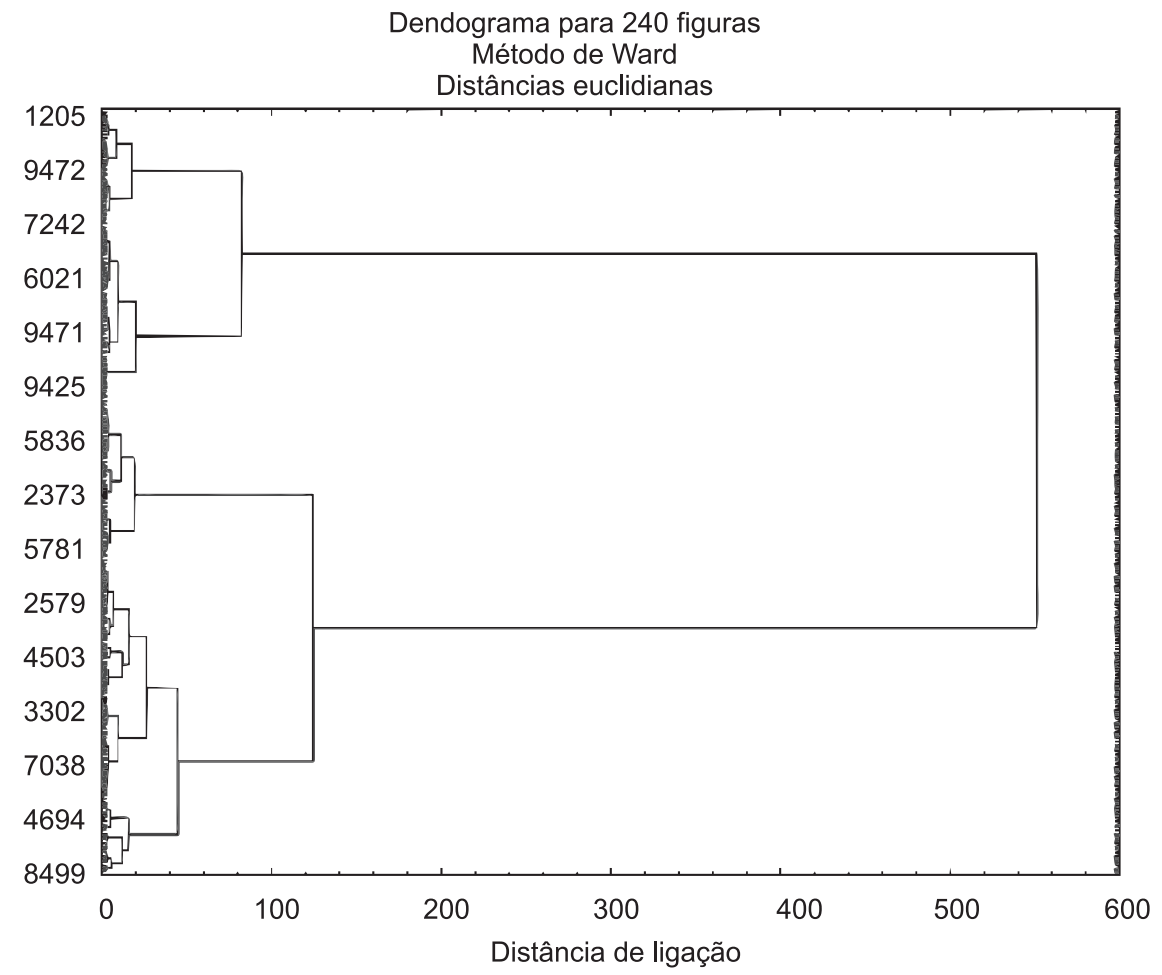

Figura 1 - Análise de cluster (seguindo o método de Ward) calculada com base nas distâncias euclidianas das médias masculinas e femininas das classificações de prazer, alerta e dominância para o total de 240 fotografias 
ETA $^{2}$ e PRE contra o número de clusters

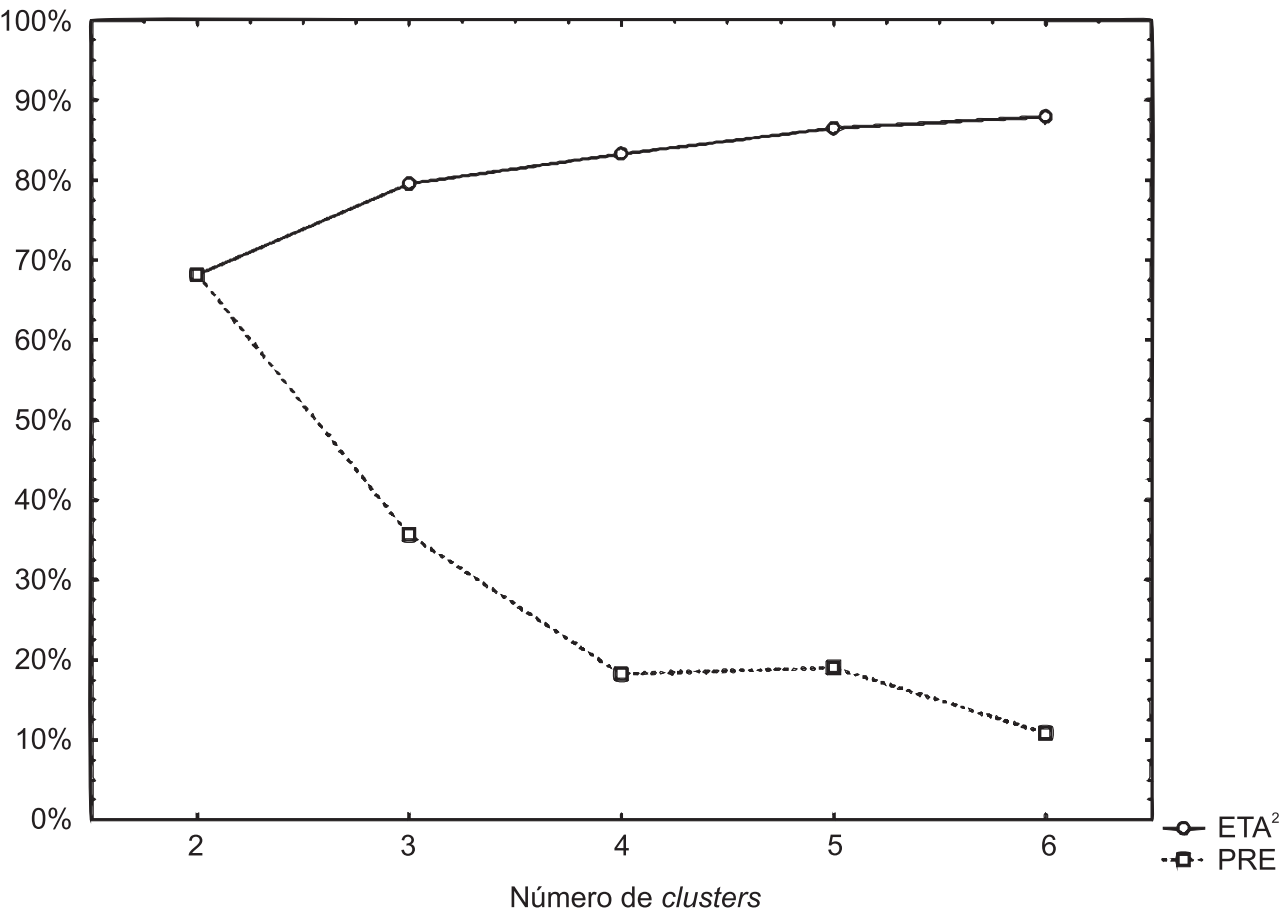

Figura 2 - Índice $\mathrm{ETA}^{2}$ de proporção de variabilidade interna explicada e índice PRE de redução proporcional de variabilidade interna em função da escolha do número de clusters

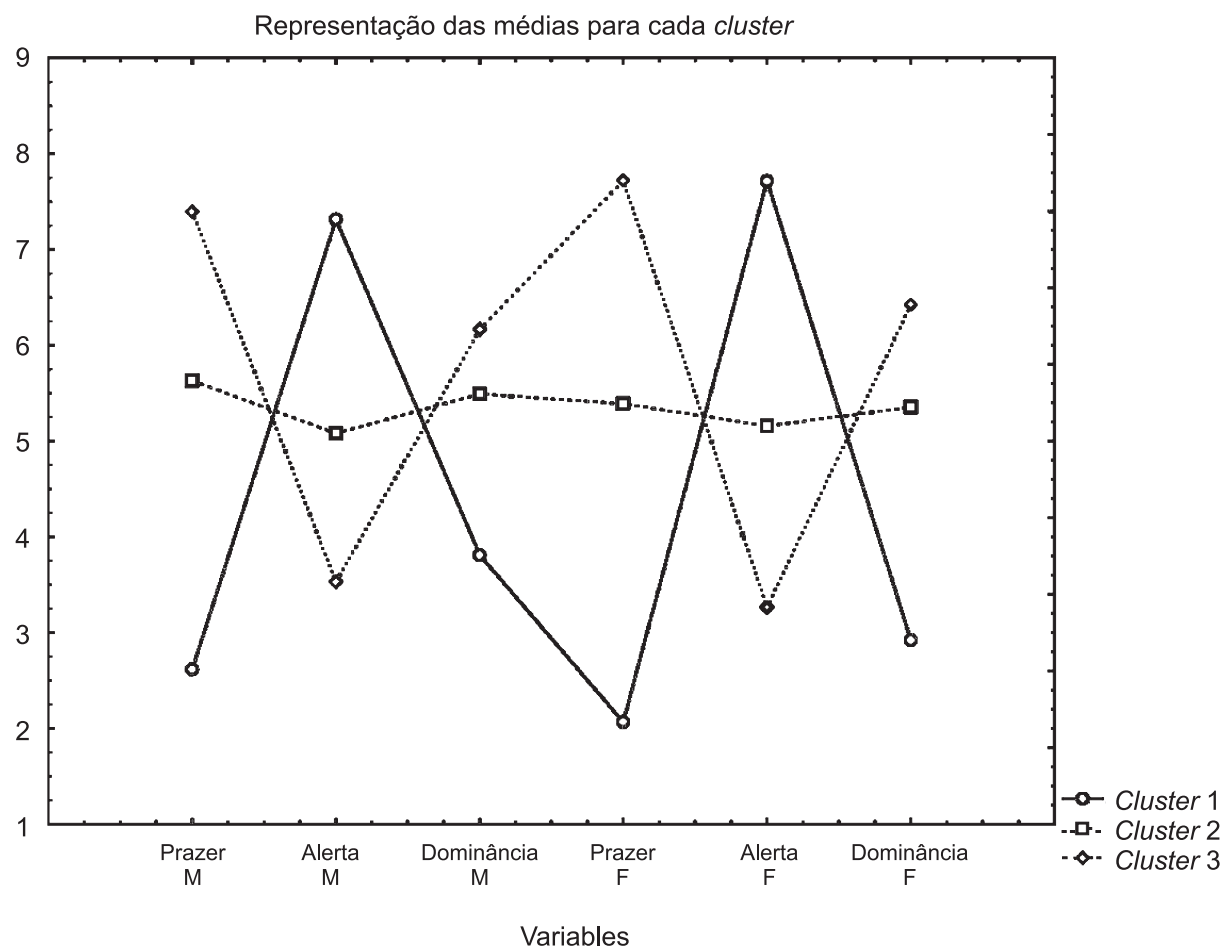

Figura 3 - Análise dos perfis dos três clusters encontrados, com base nas médias masculinas e femininas de prazer, alerta e dominância das fotografias: cluster 1 - figuras desagradáveis $(n=79)$; cluster 2 - figuras neutras $(n=97)$; cluster 3 - figuras agradáveis $(n=64)$. 


\section{Discussão}

As avaliações afetivas foram diferentes para homens e mulheres quanto às figuras classificadas como desagradáveis, para as quais as mulheres atribuíram maiores valores de alerta e menores valores de prazer e dominância. Mulheres, quando comparadas aos homens, classificaram mais figuras como agradáveis e desagradáveis, e menos como neutras, o que se deve, possivelmente, a uma percepção mais acentuada da valência emocional, que também se reflete no nível de alerta, que é altamente correlacionado ao prazer. Ademais, esse padrão diferencial de resposta entre os sexos também foi descrito nas normatizações norte-americana e espanhola do IAPS ${ }^{1,6,7}$, e é consistente com outros achados da literatura ${ }^{10}$. Esses dados corroboram a observação de que a memória emocional, bem como a experiência subjetiva das emoções, é mais marcante nas mulheres, o que pode explicar a maior incidência de distúrbios depressivo-ansiosos na população feminina.

Nossa análise também verificou que os estímulos do IAPS podem ser agrupados em três clusters, confirmando a validade das três categorias de imagens descritas (desde o estudo anterior ${ }^{8,9}$ ) como "agradáveis", "neutras" e "desagradáveis", e demonstrando que essas categorias de agradabilidade não dependem exclusivamente da valência emocional, mas são influenciadas por outros fatores relevantes na experiência das emoções, como o nível de alerta e a dominância.

Dentre as variadas técnicas de indução de estados afetivos hoje disponíveis para uso em pesquisa, o IAPS é um dos instrumentos mais usados no mundo, sendo, contudo, ainda pouco conhecido e raramente utilizado no Brasil. Pareado com o SAM, o IAPS é uma ferramenta de avaliação emocional muito versátil, simples de ser aplicada e fácil de ser compreendida, possibilitando seu uso em uma grande diversidade de sujeitos experimentais, e, também, por permitir uma coleta de dados eficiente, baseada em uma análise tridimensional das emoções, com potencial para explorar aspectos elaborados da motivação e do comportamento, como valência afetiva, alerta emocional e dominância. Por essas razões, é importante disponibilizar os valores normativos do IAPS para a população brasileira a todos os pesquisadores e profissionais interessados, para a condução de pesquisas na área da cognição, da emoção e dos distúrbios neuropsiquiátricos.
Concluindo, este estudo expande a adaptação brasileira do IAPS, acrescentando os valores normativos de 240 novos estímulos e confirmando sua validade experimental dentro de categorias emocionais pré-estabelecidas. Os valores obtidos mostram excelente estabilidade com a primeira parte da padronização brasileira e boa consistência com os dados da padronização original, reforçando a confiabilidade do IAPS como um instrumento psicométrico.

\section{Agradecimentos}

À FAPESP (Projeto $n^{0}$ 2006/05144-7) e à AFIP pelo suporte financeiro; aos professores e estudantes das universidades UNICID, UNIFESP, UniFMU, PUC, São Luís, São Marcos, Faculdades Paulistanas (FAPA) e Fundação Escola de Comércio Álvares Penteado (FECAP), que nos assistiram na coleta dos dados.

\section{Referências}

1. Lang PJ, Bradley MM, Cuthbert BN. International Affective Picture System (IAPS): Affective ratings of pictures and instruction manual. Technical Report A-6. Gainesville: University of Florida; 2005. http://www.unifesp.br/dpsicobio/adap/instructions.pdf. Acessado $\operatorname{dez} 2008$.

2. Mehrabian A, Russel JA. An approach to enviromental psychology. Cambridge: MIT; 1974.

3. Bradley MM, Codispoti M, Cuthbert BN, Lang PJ. Emotion and motivation $\mathrm{I}$ : defensive and appetitive reactions in picture processing. Emotion. 2001;1(3):276-98.

4. Ribeiro RL, Teixeira-Silva F, Pompéia S, Bueno OFA. IAPS includes photographs that elicit low-arousal physiological responses in healthy volunteers. Physiol Behav. 2007;91(5):671-5.

5. Lang PJ. Behavioral treatment and behavioral assessment: computer applications. In: Sidowski JB, Johnson JH, Williams TA, eds. Technology in mental health care delivery systems. Norwood: Ablex; 1980. p. 119-37.

6. Moltó J, Montañés S, Poy R, Segarra P, Pastor MC, Torno MP, et al. Un nuevo método para el estudio experimental de las emociones: Int Affect Pict Syst (IAPS): Adaptación Española. Rev Psicol Gen Aplicada. 1999;52(1):55-87.

7. Vila S, Sánchez M, Ramírez I, Fernández MC, Cobos P, Rodríguez $\mathrm{S}$, et al. El Sistema Internacional de Imágenes Afectivas (IAPS). Adaptación española. Segunda Parte. Rev Psicol Gen Aplicada. 1999;54(4):635-57.

8. Ribeiro RL, Pompéia S, Bueno OFA. Brazilian norms for the International Affective Picture System (IAPS): brief report. Rev Psiquiatr RS. 2004;26(2):190-4.

9. Ribeiro RL, Pompéia S, Bueno OFA. Comparison of Brazilian and North-American Norms for the International Affective Picture System (IAPS). Rev Bras Psiquiatr. 2005;27:208-15. http:// www.scielo.br/pdf/rbp/v27n3/a09v27n3.pdf. Acessado dez 2008.

10. Canli T, Desmond JE, Zhao Z, Gabriel JD. Sex differences in the neural basis of emotional memories. Proc Natl Acad Sci USA. 2002;99(16):10789-94. 